\title{
ON SUBGROUPS RELATED TO THE TENSOR CENTER
}

\author{
DAVID P. BIDDLE \\ Department of Mathematics, Cornell University, Ithaca, NY 14853-4201, USA \\ e-mail: biddle@math.cotnell.edu \\ and LUISE-CHARLOTTE KAPPE \\ Department of Mathematical Sciences, SUNY at Binghamton, Binghamton, NY 13902-6000, USA \\ e-mail:menger@math.binghamton.edu
}

(Received 8 April, 2002; accepted 8 August, 2002)

\begin{abstract}
The tensor center of a group $G$ is the set of elements $a$ in $G$ such that $a \otimes g=1_{\otimes}$ for all $g$ in $G$. It is a characteristic subgroup of $G$ contained in its center. We introduce tensor analogues of various other subgroups of a group such as centralizers and 2-Engel elements and investigate their embedding in the group as well as interrelationships between those subgroups.
\end{abstract}

2000 Mathematics Subject Classification. 20F99.

1. Introduction. The nonabelian tensor square of a group is a special case of the nonabelian tensor product which has its origins in algebraic $K$-theory as well as in homotopy theory. It is defined as follows.

DEFINITION 1.1. For a group $G$, the nonabelian tensor square $G \otimes G$ is generated by the symbols $g \otimes h, g, h \in G$, subject to the relations

$$
\begin{aligned}
& g g^{\prime} \otimes h=\left({ }^{g} g^{\prime} \otimes^{g} h\right)(g \otimes h), \\
& g \otimes h h^{\prime}=(g \otimes h)\left({ }^{h} g \otimes{ }^{h} h^{\prime}\right),
\end{aligned}
$$

for all $g, g^{\prime}, h, h^{\prime} \in G$, where ${ }^{h} g=h g h^{-1}$ denotes the conjugate of $g$ by $h$.

Everyone agrees that some ideas leading to this concept can already be found in Whitehead's work [15]; e.g. the universal quadratic function $\Gamma$. The nonabelian tensor square appears in essence but not in name in the work of Keith Dennis and is based on ideas of C. Miller [14]. Independently, Lue in [12] defines nonabelian tensor products in the setting of nilpotent groups, extending earlier work by Ganea [7]. As an outgrowth of their involvement with generalized Van Kampen theorems, Brown and Loday in [3] and [4] introduce nonabelian tensor products, establish their topological significance and start the investigation of these products as group theoretical objects. In [2] this work is continued with focus on nonabelian tensor squares. In the wake of [2], many papers have appeared, mainly concerned with the explicit computation of nonabelian tensor squares. For an overview of these results we refer to [9]. For further details on the subject we refer the interested reader to the following web site:

http://www.bangor.ac.uk/ mas010/nonabtens.html

The topic of this paper is the investigation of subgroups of a group defined via its nonabelian tensor square, modeled after the tensor center $Z^{\otimes}(G)$ of a group $G$, 
introduced by Ellis [6] as the set of all $a \in G$ with $a \otimes x=1_{\otimes}$, for all $x \in G$, with $1_{\otimes}$ being the identity of $G \otimes G$. The starting point of our investigations is the following generalization of the tensor center.

Definition 1.2. For a group $G$ and a non-empty subset $X$ we define the tensor annihilator of $X$ in $G$ to be the set

$$
C^{\otimes}(X)=\left\{a \in G \mid a \otimes x=1_{\otimes} \forall x \in X\right\} .
$$

The tensor annihilator is an analogue of the centralizer of a subset $X$ of elements in a group $G$; i.e. $C_{G}(X)=\{a \in G \mid[a, x]=1 \forall x \in X\}$, where $[g, h]=g h g^{-1} h^{-1}$, the commutator of $g, h \in G$. As we shall see in the third section, results analogous to those for centralizers in a group will hold for tensor annihilators. In [6], it was shown that $Z^{\otimes}(G)$ is a characteristic subgroup of $G$ contained in $Z(G)$, the center of $G$. This result is a corollary to our results on tensor annihilators, since $Z^{\otimes}(G)=C^{\otimes}(X)$ for $X=G$.

An alternative characterization of the center of a group as the margin of the commutator word gives rise to an analogue in the setting of nonabelian tensor products. Margins were introduced by P. Hall in [8]. Let $\varphi\left(x_{1}, \ldots, x_{n}\right)$ be a word in the variables $x_{1}, \ldots, x_{n}$. The $i$-th partial margin, $1 \leq i \leq n$, of $\varphi$ in a group $G$ is the set of all $a \in G$ with $\varphi\left(g_{1}, \ldots, a g_{i}, \ldots, g_{n}\right)=\varphi\left(g_{1}, \ldots, g_{i}, \ldots, g_{n}\right)$ for all $g_{1}, \ldots, g_{n} \in G$, denoted by $\varphi_{i}^{*}(G)$. The margin $\varphi^{*}(G)$ of the word $\varphi$ is defined as the intersection of the partial margins $\varphi_{i}^{*}(G), i=1, \ldots, n$. Margins and partial margins are characteristic subgroups of the group.

We have $\gamma_{1}^{*}(G)=\{a \in G \mid[a g, h]=[g, h] \forall g, h \in G\}$, where $\gamma(x, y)=[x, y]$ is the commutator word and it can be easily seen that $\gamma_{1}^{*}(G)=\gamma_{2}^{*}(G)=\gamma^{*}(G)=Z(G)$. In [10], a local version of a margin was introduced for various 2-variable commutator words $\varphi(x, y)$ as the set of all $a$ such that $\varphi(a x, g)=\varphi(x, g)$, for fixed $g$ and all $x \in G$; the properties of these sets and the interrelationship between them and the respective margins were investigated. It is natural to ask whether there are tensor analogues for margins and its localized version, in particular for the commutator word $\gamma(x, y)=$ $[x, y]$. This is the topic of our investigations in Section 4 of this paper, where we consider the set of all $a \in G$ such that $a x \otimes y=x \otimes y$, for all $x, y \in G$ and all $b \in G$ such that $x b \otimes g=x \otimes g$, for fixed $g$ and all $x \in G$, respectively. As we shall find out, the tensor analogues share many properties with the original group concepts.

The question arises whether there exist tensor analogues for other commutator words such as normed commutators of weight $n$; namely $\gamma\left(x_{1}, \ldots, x_{n}\right)=$ $\left[x_{1}, \ldots, x_{n}\right]$, where $\left[x_{1}, \ldots, x_{n-1}, x_{n}\right]=\left[\left[x_{1}, \ldots, x_{n-1}\right], x_{n}\right]$, and the $n$-Engel word $\epsilon_{n}(x, y)=\left[x,{ }_{n} y\right]$ where $\left[x,{ }_{n} y\right]=\left[\left[x,{ }_{n-1} y\right], y\right]$ and $\left[x,{ }_{1} y\right]=[x, y]$. For normed commutators of weight $n$ we have

$$
Z_{n}(G)=\left\{a \in G \mid\left[a, g_{2}, \ldots, g_{n}\right]=1 \forall g_{2}, \ldots, g_{n} \in G\right\},
$$

the $n$-th center of the group $G$, which is characteristic in $G$. It can be easily seen that

$$
Z_{n}^{\otimes}(G)=\left\{a \in G \mid\left[a, g_{2}, \ldots, g_{n-1}\right] \otimes g_{n}=1_{\otimes} \forall g_{2}, \ldots, g_{n} \in G\right\}
$$

is a characteristic subgroup of $G$ contained in $Z_{n}(G)$ and it will therefore be called the $n$-th tensor center of $G$.

In contrast to the $n$-th center, the set of right $n$-Engel elements of a group $G$,

$$
R_{n}(G)=\left\{a \in G \mid\left[a,{ }_{n} g\right]=1 \forall g \in G\right\},
$$


is not necessarily a subgroup for $n \geq 3$; (see [13]). In [11], however, it was shown that $R_{2}(G)=\{a \in G \mid[a, g, g]=1 \forall a \in G\}$ is a characteristic subgroup of $G$. Tensor analogues of $R_{n}(G)$ can be easily defined as

$$
R_{n}^{\otimes}(G)=\left\{a \in G \mid\left[a,{ }_{n-1} g\right] \otimes g=1_{\otimes} \forall g \in G\right\} .
$$

In the last section of this paper we shall show that $R_{2}^{\otimes}(G)$ is a characteristic subgroup of $G$, containing $Z(G)$ and contained in $R_{2}(G)$. We conclude this section with an example of a group $W$ in which the containments $Z^{\otimes}(W)<Z(W)<R_{2}^{\otimes}(W)<R_{2}(W)$ are all proper.

2. Basic results. This section contains basic results on nonabelian tensor squares, as can be found in [2], and some lemmas to be used throughout the rest of the paper. We start with the familiar commutator expansion formulas when left action is used, whose similarities to the defining relations of the nonabelian tensor square do not come as a surprise since the properties of $G \otimes G$ are related to the biderivation property of commutators.

LEMMA 2.1. Let $G$ be a group and $g, g^{\prime}, h, h^{\prime} \in G$ with $[g, h]=g h g^{-1} h^{-1}$, the commutator of $g$ and $h$. Then

$$
\begin{aligned}
& {\left[g g^{\prime}, h\right]=\left[{ }^{g} g^{\prime},{ }^{g} h\right][g, h],} \\
& {\left[g, h h^{\prime}\right]=[g, h]\left[{ }^{h} g,{ }^{h} h^{\prime}\right] .}
\end{aligned}
$$

In our last section we discuss the tensor analogue of right 2-Engel elements. The following formulae which hold for these elements are of importance in this context.

Proposition 2.2. [11] Let $G$ be a group and $R_{2}(G)=\{a \in G \mid[a, g, g]=1 \forall g \in G\}$. Then the following hold for $a, b \in R_{2}(G)$ and $g, h \in G$ :

$$
\begin{aligned}
{[[a, g],[h, g]] } & =1 ; \\
{[a, g, h, h] } & =1 ; \\
{[a, g, h][a, h, g] } & =1 ; \\
{[a, g, a] } & =1 ; \\
{[[a, g],[a, h]] } & =1 .
\end{aligned}
$$

Proof. The first four identities follow immediately from [11] and (2.2.5) is a consequence of the fact that the normal closure of $a \in R_{2}(G)$ is abelian, as shown in [11].

The group $G$ acts on $G \otimes G$ by ${ }^{g}(u \otimes v)={ }^{g} u \otimes{ }^{g} v$, for $g, u, v \in G$. With this observation, the following result is a consequence of Definition 1.1. For the interested reader we mention that a key ingredient of the proof of this proposition is expansion of $g h \otimes g^{\prime} h^{\prime}$ in two ways, a widely used technique in commutator calculus.

Proposition 2.3. [2, Proposition 3]. The following relations hold for all $g, g^{\prime}, h$, $h^{\prime} \in G$ : 


$$
\begin{gathered}
{ }^{g}\left(g^{-1} \otimes h\right)=(g \otimes h)^{-1}={ }^{h}\left(g \otimes h^{-1}\right), \\
(g \otimes h)\left(g^{\prime} \otimes h^{\prime}\right)(g \otimes h)^{-1}={ }^{[g, h]}\left(g^{\prime} \otimes h^{\prime}\right), \\
{[g, h] \otimes h^{\prime}=(g \otimes h){ }^{h^{\prime}}(g \otimes h)^{-1},} \\
g^{\prime} \otimes[g, h]={ }^{g^{\prime}}(g \otimes h)(g \otimes h)^{-1}, \\
{\left[g \otimes h, g^{\prime} \otimes h^{\prime}\right]=[g, h] \otimes\left[g^{\prime}, h^{\prime}\right] .}
\end{gathered}
$$

The next result is the key to the striking analogies between the tensor annihilator and the centralizer of a subset $X$ in a group $G$.

Proposition 2.4. [2, Proposition 2]. Let $G$ be a group and $G^{\prime}=\langle[g, h] \mid g, h \in G\rangle$ its commutator subgroup. Then there exists a homomorphism $\kappa: G \otimes G \rightarrow G^{\prime}$ such that $\kappa(g \otimes h)=[g, h]$. Furthermore, ker $\kappa$, denoted by $J_{2}(G)$, is a central subgroup of $G \otimes G$ and $G$ acts trivially on $J_{2}(G)$.

Proposition 2.5. [2, Proposition 11]. Let $U, V$ be groups. Then

$$
(U \times V) \otimes(U \times V) \cong(U \otimes U) \times(V \otimes V) \times\left(U_{a b} \otimes_{\mathbb{Z}} V_{a b}\right) \times\left(V_{a b} \otimes_{\mathbb{Z}} U_{a b}\right),
$$

where $U_{a b}$ and $V_{a b}$ are the abelianizations of $U$ and $V$, respectively, and $\otimes_{\mathbb{Z}}$ denotes the abelian tensor over $\mathbb{Z}$.

Proposition 2.6. [2, Proposition 9] Given a central extension $1 \rightarrow A \rightarrow K \stackrel{\pi}{\rightarrow}$ $G \rightarrow 1$, then there is an exact sequence $(A \otimes K) \times(K \otimes A) \stackrel{\iota}{\rightarrow} K \otimes K \stackrel{\pi \otimes \pi}{\longrightarrow} G \otimes G \rightarrow 1$ in which Im เ is central.

For groups of class two the tensor expansion formulas reduce to the following.

Lemma 2.7. [1] Let $G$ be a group of class 2 and $a, b, c, d, e \in G$. Then

$$
\begin{aligned}
{[a, b][c, d] \otimes e } & =([a, b] \otimes e)([c, d] \otimes e), \\
{[a, b] \otimes c d } & =([a, b] \otimes c)([a, b] \otimes d)
\end{aligned}
$$

3. Tensor annihilators. We start with the main result of this section, a theorem which details the properties of the tensor annihilator of a set $X$. It is the exact analogue of the theorem which holds for the centralizer of a subset $X$ of a group $G$. Just replacing $C^{\otimes}(X)$ by $C_{G}(X)$ gives the familiar result for groups. Similarly, as we defined an action of the group $G$ on its tensor square $G \otimes G$, we can define an action of $\operatorname{Aut}(G)$, the automorphism group of $G$, on $G \otimes G$ as follows. For $\alpha \in A u t(G)$, let ${ }^{\alpha}(g \otimes h)={ }^{\alpha} g \otimes{ }^{\alpha} h$ for $g, h \in G$.

THEOREM 3.1. Let $G$ be a group and $X$ a non-empty subset of $G$. Then

$$
C^{\otimes}(X)=\left\{a \in G \mid a \otimes x=1_{\otimes} \forall x \in X\right\},
$$

the tensor annihilator of $X$ in $G$, is a subgroup of $G$ contained in $C_{G}(X)$. If $X$ is a normal subset or a characteristic subset of $G$, then $C^{\otimes}(X)$ is a normal or characteristic subgroup, respectively. 
Proof. Let $a \in C^{\otimes}(X)$. Then, by Proposition 2.4, $\kappa(a \otimes x)=[a, x]=1$, for all $x \in X$. Hence $C^{\otimes}(X)$ is contained in $C_{G}(X)$. For $1_{G}$, the identity of $G$, we have $1_{G} \otimes x=$ $1_{\otimes}$ for all $x \in X$. Hence $C^{\otimes}(X) \neq \emptyset$. Now let $a, b \in C^{\otimes}(X)$. Then, by (1.1.1) and (2.3.1), we have $a b^{-1} \otimes x={ }^{a}\left(b^{-1} \otimes x\right)(a \otimes x)={ }^{a b^{-1}}(b \otimes x)^{-1}(a \otimes x)=1_{\otimes}$ for all $x \in X$; hence $a b^{-1} \in C^{\otimes}(X)$. We conclude that $C^{\otimes}(X)$ is a subgroup of $G$.

Now let $X$ be a characteristic subset of $G$; i.e. ${ }^{\alpha} x \in X$, for all $\alpha \in \operatorname{Aut}(G)$ and all $x \in X$. Then, for $a \in C^{\otimes}(X)$, we have ${ }^{\alpha} a \otimes x={ }^{\alpha}\left(a \otimes \alpha^{\alpha^{-1}} x\right)=1_{\otimes}$, for all $x \in X$ and all $\alpha \in \operatorname{Aut}(G)$. It follows that $C^{\otimes}(X)$ is a characteristic subgroup of $G$. The proof that $C^{\otimes}(X) \triangleleft G$ if $X$ is a normal subset of $G$ is similar and therefore omitted.

As is customary, we write $C^{\otimes}(g)$ instead of $C^{\otimes}(\{g\})$ if $X=\{g\}$, and call $C^{\otimes}(g)$ the tensor centralizer of $g$ in $G$. Observe that, contrary to the group centralizer, the element $g$ is not necessarily contained in $C^{\otimes}(g)$. The following proposition is now immediate.

Proposition 3.2. Let $G$ be a group and $X$ a non-empty subset of $G$. Then

$$
C^{\otimes}(X)=\bigcap_{x \in X} C^{\otimes}(x)=C^{\otimes}(\langle X\rangle)
$$

Proof. The first part of our claim follows from the definition of $C^{\otimes}(X)$. For the second part observe that obviously $C^{\otimes}(\langle X\rangle) \subseteq C^{\otimes}(X)$. Now consider $a \in C^{\otimes}(X)$ and $u \in\langle X\rangle$, where $u$ is a word in $X \cup X^{-1}$. It follows by (1.1.2), (2.2.1), and induction on the length of the word $u$ that $a \otimes u=1_{\otimes}$. We conclude that $C^{\otimes}(X) \subseteq C^{\otimes}(\langle X\rangle)$.

The result by Ellis in [6] and the tensor analogue of the fact that the center of the group is the intersection of all centralizers is now an immediate consequence of Theorem 3.1 and Proposition 3.2.

COROLlaRY 3.3. Let $G$ be a group. Then the tensor center $Z^{\otimes}(G)$ is a characteristic subgroup of $G$ contained in the center of $G$. Furthermore, $Z^{\otimes}(G)=\bigcap_{g \in G} C^{\otimes}(g)$.

For use in the next section, we state here another immediate corollary of Theorem 3.1 and Proposition 3.2.

COROLlARY 3.4. Let $G$ be a group and ${ }^{G} g=\left\langle{ }^{x} g \mid x \in G\right\rangle$, the normal closure of $g$ in $G$. Then $C^{\otimes}\left({ }^{G} g\right)$ is a normal subgroup of $G$ contained in $C_{G}\left({ }^{G} g\right)$ and $C^{\otimes}\left({ }^{G} g\right)=\bigcap_{x \in G} C^{\otimes}\left({ }^{x} g\right)$.

4. Tensors and margins. In this section we explore a tensor analogue of P. Hall's margins in the case of the commutator word [8]. Our investigations are guided by the Model Proposition stated in [10]. To make our notions more precise, we give the following definitions using left action.

Definition 4.1. Let $G$ be a group and $g \in G$. Then

$$
\begin{gathered}
{ }^{*} E(G, g)=\{a \in G \mid[x a, g]=[x, g] \forall x \in G\}, \\
E^{*}(G, g)=\{a \in G \mid[a x, g]=[x, g] \forall x \in G\} .
\end{gathered}
$$

(Note that in this context, the left and right asterisks denote absorption to the left and right, respectively, whereas for right action as used in [10], left and right asterisks 
denote absorption from the left and right, respectively.) In [10], it was shown that ${ }^{*} E(G, g)$ and $E^{*}(G, g)$ are subgroups and $\bigcap_{g \in G} E^{*}(G, g)=\bigcap_{g \in G} * E(G, g)=Z(G)$.

We are now ready to formulate the proposition from [10] of which we shall prove a tensor analogue.

Model Proposition. [10] Let $G$ be a group, $g \in G$, and ${ }^{G} g$ the normal closure of $g$ in $G$. Then

(i) ${ }^{*} E(G, g)=C_{G}(g)$,

(ii) $E^{*}(G, g)=\bigcap_{x \in G} C_{G}\left({ }^{x} g\right)=C_{G}\left({ }^{G} g\right)$,

(iii) $E^{*}(G, g) \triangleleft G$ and $E^{*}(G, g) \subseteq{ }^{*} E(G, g)$.

We shall now define the tensor analogues of ${ }^{*} E(G, g)$ and $E^{*}(G, g)$.

Definition 4.2. Let $G$ be a group and $g \in G$. Then

$$
\begin{aligned}
& { }^{\otimes} E(G, g)=\{a \in G \mid x a \otimes g=x \otimes g \forall x \in G\}, \\
& E^{\otimes}(G, g)=\{a \in G \mid a x \otimes g=x \otimes g \forall x \in G\} .
\end{aligned}
$$

With these definitions we can now state and prove the tensor analogue of the Model Proposition.

THEOREM 4.3. Let $G$ be a group, $g \in G$, and ${ }^{G} g$ the normal closure of $g$ in $G$. Then ${ }^{\otimes} E(G, g)$ and $E^{\otimes}(G, g)$ are subgroups of $G$. Also we have

$$
\begin{aligned}
{ }^{\otimes} E(G, g) & =C^{\otimes}(g), \\
E^{\otimes}(G, g) & =C^{\otimes}\left({ }^{G} g\right), \\
E^{\otimes}(G, g) \triangleleft G \text { and } E^{\otimes}(G, g) & \subseteq{ }^{\otimes} E(G, g) .
\end{aligned}
$$

Proof. The fact that ${ }^{\otimes} E(G, g)$ and $E^{\otimes}(G, g)$ are subgroups follows immediately from (4.3.1) and (4.3.2) as well as Theorem 3.1 for $X=\{g\}$ and Corollary 3.4, respectively. To prove (4.3.1) we observe that by (1.1.1) we have $x a \otimes g={ }^{x}(a \otimes g)(x \otimes g)$ for $a, g, x \in G$. By (4.2.1) and Definition 1.2, it follows that $a \in{ }^{\otimes} E(G, g)$ if and only if $a \in C^{\otimes}(g)$. Thus (4.3.1) follows.

To prove (4.3.2), we observe that by (1.1.1) and (2.3.1) we have

$$
x^{-1}\left(a \otimes{ }^{x} g\right)=x^{-1}(a x) \otimes g=x^{-1}\left((a x \otimes g)(x \otimes g)^{-1}\right) .
$$

Thus $a \otimes{ }^{x} g=1_{\otimes}$ if and only if $a x \otimes g=x \otimes g$. We conclude that $E^{\otimes}(G, g)=C^{\otimes}\left({ }^{G} g\right)$.

By Corollary 3.4 together with (4.3.2), we have $E^{\otimes}(G, g) \triangleleft G$, and $C^{\otimes}\left({ }^{G} g\right) \leq C^{\otimes}(g)$ together with (4.3.1) and (4.3.2) implies that $E^{\otimes}(G, g) \subseteq{ }^{\otimes} E(G, g)$, proving (4.3.3.)

The following analogue of Proposition 1.2 in [10] is now an immediate consequence of Theorem 4.3 and Corollaries 3.3 and 3.4.

COROLlaRY 4.4. Let $G$ be a group. Then $Z^{\otimes}(G)=\bigcap_{g \in G} E^{\otimes}(G, g)=\bigcap_{g \in G}^{\otimes} E(G, g)$.

5. The tensor analogue of right 2-Engel elements. In this section we shall show that $R_{2}^{\otimes}(G)=\left\{a \in G \mid[a, g] \otimes g=1_{\otimes} \forall g \in G\right\}$ is a characteristic subgroup of $G$. This is the tensor analogue of the fact that the set of right 2-Engel elements, i.e. $R_{2}(G)=$ 
$\{a \in G \mid[[a, g], g]=1 \forall g \in G\}$, is a characteristic subgroup of $G$, as shown in [11]. We start with a few lemmas.

LEMMA 5.1. Let $G$ be a group and $a \in R_{2}^{\otimes}(G), g, h \in G$. Then

$$
\begin{aligned}
(a \otimes g)^{-1} & =a \otimes g^{-1}, \\
([a, h] \otimes g)([a, g] \otimes h) & =1_{\otimes} .
\end{aligned}
$$

Proof. To show (5.1.1) we observe that $[a, g] \otimes g=1_{\otimes}$ implies $^{g}(a \otimes g)=a \otimes g$ by (2.3.3). By (2.3.1), this yields $a \otimes g=g^{-1}(a \otimes g)=g^{-1}\left({ }^{g}\left(a \otimes g^{-1}\right)^{-1}\right)=\left(a \otimes g^{-1}\right)^{-1}$. We conclude that (5.1.1) holds.

To show (5.1.2), we observe that by (5.1.1) we have $a \otimes g h=\left(a \otimes(g h)^{-1}\right)^{-1}$. Expanding both sides by (1.1.2) and observing (5.1.1) we arrive at $(a \otimes g) \cdot{ }^{g}(a \otimes h)=$ $h^{-1}(a \otimes g)(a \otimes h)$, or equivalently, after rearranging and inverting, we obtain

$$
\left((a \otimes h) \cdot{ }^{g}(a \otimes h)^{-1}\right)^{-1}=\left(a \otimes g^{-1}\right) \cdot h^{-1}\left(a \otimes g^{-1}\right)^{-1} .
$$

By (2.3.3) it follows that

$$
([a, h] \otimes g)^{-1}=\left[a, g^{-1}\right] \otimes h^{-1} .
$$

Using (2.3.1), we obtain for the right side of the above that

$$
\left[a, g^{-1}\right] \otimes h^{-1}={ }^{[a, g]}\left([a, g] \otimes h^{-1}\right)^{-1} .
$$

Substituting this into (5.1.3) yields after inversion

$$
([a, h] \otimes g) \cdot{ }^{[a, g]}\left([a, g] \otimes h^{-1}\right)^{-1}=1_{\otimes} .
$$

Conjugating the above by $[g, a]$, using (2.2.1) and (2.3.1) yields

$$
([a, h] \otimes g)\left({ }^{h}[a, g] \otimes h\right)=1_{\otimes} .
$$

After substituting $h^{-1}$ for $h$ into the above we arrive at

$$
\left([a, h]^{-1} \otimes g\right)\left(h^{-1}[a, g] \otimes h^{-1}\right)=1_{\otimes} .
$$

Using (2.3.1), conjugating with $[h, a]$, observing (2.2.1) and inversion lead to

$$
([a, g] \otimes h)([a, h] \otimes g)=1_{\otimes},
$$

the desired result.

LemmA 5.2. Let $a \in R_{2}^{\otimes}(G)$ and $g, h \in G$. Then ${ }^{g}[a, h] \otimes h=1_{\otimes}$.

Proof. Expanding $1_{\otimes}=[a, g h] \otimes g h$ by (2.1.2) and (1.1.2), we arrive at

$$
1_{\otimes}=\left([a, g] \cdot{ }^{g}[a, h] \otimes g\right) \cdot{ }^{g}\left([a, g] \cdot{ }^{g}[a, h] \otimes h\right) .
$$

Further expansion using (1.1.1) leads to

$$
1_{\otimes}=\left[{ }^{[a, g]}\left({ }^{g}[a, h] \otimes g\right) \cdot{ }^{g}\left({ }^{[a, g]}\left({ }^{g}[a, h] \otimes h\right) \cdot([a, g] \otimes h)\right) .\right.
$$


Observing that $[a, g]$ acts trivially on the first factor and conjugating by $g^{-1}$, we obtain

$$
1_{\otimes}=([a, h] \otimes g) \cdot{ }^{[a, g]}\left({ }^{g}[a, h] \otimes h\right) \cdot([a, g] \otimes h) .
$$

Note that $\kappa\left({ }^{g}[a, h] \otimes h\right)=\left[{ }^{g}[a, h], h\right]=[g,[a, h], h]=1$, by (2.2.2) and (2.2.3). Thus ${ }^{g}[a, h] \otimes h \in \operatorname{ker} \kappa$ and $[a, g]$ acts trivially on it. Hence (5.2.1) reduces to

$$
1_{\otimes}=([a, h] \otimes g) \cdot\left({ }^{g}[a, h] \otimes h\right) \cdot([a, g] \otimes h) .
$$

Finally, by using (5.1.2), the equation above reduces to $1_{\otimes}={ }^{g}[a, h] \otimes h$, the desired result.

Now we are in a position to prove the main result of this section.

THEOREM 5.3. Let $G$ be a group and $R_{2}^{\otimes}(G)=\left\{a \in G \mid[a, g] \otimes g=1_{\otimes} \forall g \in G\right\}$. Then $R_{2}^{\otimes}(G)$ is a characteristic subgroup of $G$ containing $Z(G)$ and being contained in $R_{2}(G)$.

Proof. The containments and the fact that $R_{2}^{\otimes}(G)$ is a characteristic set are both obvious. It remains to be shown that $R_{2}^{\otimes}(G)$ is closed under inverses and products.

If $a \in R_{2}^{\otimes}(G)$, then, by (2.2.4), (2.3.1), and the fact that $R_{2}^{\otimes}(G) \subseteq R_{2}(G)$, we obtain

$$
\left[a^{-1}, g\right] \otimes g=[a, g]^{-1} \otimes g=([a, g] \otimes g)^{-1}=1_{\otimes} .
$$

Hence $a^{-1} \in R_{2}^{\otimes}(G)$. Now let $a, b \in R_{2}^{\otimes}(G)$ and $g \in G$. Then, by expansion using (2.1.1) and (1.1.1), we arrive at $[a b, g] \otimes g={ }^{a}[b, g] \otimes g$. It follows that $[a b, g] \otimes g=1_{\otimes}$ by Lemma 5.2. Thus $a b \in R_{2}^{\otimes}(G)$, the desired result.

We conclude this paper with an example of a group in which $R_{2}^{\otimes}$ is properly contained in the set of right 2-Engel elements and the center is properly contained in $R_{2}^{\otimes}$.

EXAMPLE 5.4. Let $W=G / N$, where $G=\left\langle x_{1}, y_{1}, z_{1}, x_{2}, y_{2}, z_{2}\right\rangle=U \times V$, with $\left\langle x_{1}, y_{1}, z_{1}\right\rangle=U$ and $\left\langle x_{2}, y_{2}, z_{2}\right\rangle=V$, where $U \cong V \cong \mathcal{H}_{3}=F_{3} / \gamma_{3}\left(F_{3}\right)$, the free group of class two and rank 3. Furthermore, let $N=\left\langle\left[x_{1}, y_{1}\right]\left[y_{2}, x_{2}\right],\left[x_{1}, z_{1}\right]\left[z_{2}, x_{2}\right]\right\rangle$, a central subgroup of $G$. Then

$$
1<Z^{\otimes}(W)<Z(W)<R_{2}^{\otimes}(W)<R_{2}(W)=W,
$$

where the containments indicated are proper.

Proof. Denoting the cosets $x_{i} N, y_{i} N$ and $z_{i} N, i=1,2$, by $a_{i}, b_{i}$ and $c_{i}$, respectively, we have $W=\left\langle a_{1}, b_{1}, c_{1}, a_{2}, b_{2}, c_{2}\right\rangle$. Since $W$ has class 2 , it follows that $R_{2}(W)=W$ and $Z(W)=\left\langle\left[a_{1}, b_{1}\right],\left[a_{1}, c_{1}\right],\left[b_{1}, c_{1}\right],\left[b_{2}, c_{2}\right]\right\rangle$.

To compute $W \otimes W$, we first determine $G \otimes G$ and then obtain $W \otimes W$ as a suitable quotient of it. Since $G \cong U \times V$, it follows from Proposition 2.5 that

$$
G \otimes G=(U \otimes U) \times(V \otimes V) \times\left(U_{a b} \otimes_{\mathbb{Z}} V_{a b}\right) \times\left(V_{a b} \otimes_{\mathbb{Z}} U_{a b}\right) .
$$

Denoting by $\mathbb{Z}^{n}$ the direct product of $n$ copies of the infinite cyclic group $\mathbb{Z}$, we have $U_{a b} \cong V_{a b} \cong \mathbb{Z}^{3}$. Since the abelian tensor is bilinear, we obtain $U_{a b} \otimes_{\mathbb{Z}} V_{a b} \cong$ $V_{a b} \otimes U_{a b} \cong \mathbb{Z}^{9}$. By [1], it follows that $U \otimes U \cong V \otimes V \cong \mathcal{H}_{3} \otimes \mathcal{H}_{3} \cong \mathbb{Z}^{17}$. Hence, with the above, we arrive at $G \otimes G \cong\left(\mathbb{Z}^{17}\right)^{2} \times\left(\mathbb{Z}^{9}\right)^{2}=\mathbb{Z}^{52}$. 
In order to apply Proposition 2.6 in computing $W \otimes W$, we determine the kernel $(G \otimes N) \times(N \otimes G)$. We observe that with $L_{1}=\left\{x_{1}, y_{1}, z_{1}\right\}$ and $L_{2}=\left\{x_{2}, y_{2}, z_{2}\right\}$, we have $N \otimes G=\left\langle\left[x_{1}, y_{1}\right]\left[y_{2}, x_{2}\right] \otimes g,\left[x_{1}, z_{1}\right]\left[z_{2}, x_{2}\right] \otimes g \mid g \in L_{1} \cup L_{2}\right\rangle$ and $G \otimes N=\langle g \otimes$ $\left[x_{1}, y_{1}\right]\left[y_{2}, x_{2}\right], g \otimes\left[x_{1}, z_{1}\right]\left[z_{2}, x_{2}\right]\left|g \in L_{1} \cup L_{2}\right\rangle$. As $[a, b] \otimes c=(a \otimes b) \cdot{ }^{c}(a \otimes b)^{-1}$ $=(c \otimes[a, b])^{-1}$, by (2.3.3) and (2.3.4), we conclude that $G \otimes N=N \otimes G$. Now, by (2.3.3) and (2.7.1) and the relations of $G$, we have

$$
\left[x_{1}, w_{1}\right]\left[w_{2}, x_{2}\right] \otimes g= \begin{cases}{\left[x_{1}, w_{1}\right] \otimes g} & \text { for } g \in L_{1}, \\ {\left[w_{2}, x_{2}\right] \otimes g} & \text { for } g \in L_{2},\end{cases}
$$

where $w_{1}=y_{1}$ or $z_{1}$ and $w_{2}=y_{2}$ or $z_{2}$, respectively. It follows that

$$
N \otimes G=\left\langle\left[x_{1}, y_{1}\right] \otimes g,\left[x_{2}, z_{2}\right] \otimes h,\left[x_{1}, z_{1}\right] \otimes g,\left[x_{2}, z_{2}\right] \otimes h \mid g \in L_{1}, h \in L_{2}\right\rangle .
$$

By [1], the generators of $N \otimes G$ are independent generators of $G \otimes G$ and we observe that $(N \otimes G) \times(G \otimes N) \leq(U \otimes U) \times(V \otimes V)$. Since $N \otimes G \cong \mathbb{Z}^{12}$, we conclude that Proposition 2.6 leads to

$$
W \otimes W \cong(G \otimes G) / I m \iota \cong \mathbb{Z}^{52-12}=\mathbb{Z}^{40} .
$$

We turn now to the computation of $Z^{\otimes}(W)$ and $R_{2}^{\otimes}(W)$. Recalling $Z(W)=$ $\left\langle\left[a_{1}, b_{1}\right],\left[a_{1}, c_{1}\right],\left[b_{1}, c_{1}\right],\left[b_{2}, c_{2}\right]\right\rangle$, we observe that $\left[b_{1}, c_{1}\right] \otimes a_{1} \neq 1_{\otimes}$ and $\left[b_{2}, c_{2}\right] \otimes a_{2} \neq$ $1_{\otimes}$. We conclude that $\left[b_{1}, c_{1}\right],\left[b_{2}, c_{2}\right] \notin Z^{\otimes}(W)$. We shall show next that $\left[a_{1}, b_{1}\right] \otimes g=$ $\left[a_{1}, c_{1}\right] \otimes g=1_{\otimes}$ for all $g \in A=\left\{a_{1}, b_{1}, c_{1}, a_{2}, b_{2}, c_{2}\right\}$. Let $g=a_{1}$. Then, by (2.7.3) and the relations of $W$, it follows that

$$
\left[a_{1}, b_{1}\right] \otimes a_{1}=\left[a_{2}, b_{2}\right] \otimes a_{1}=\left(a_{2} \otimes b_{2}\right) \cdot{ }^{a_{1}}\left(a_{2} \otimes b_{2}\right)^{-1}=1_{\otimes}
$$

and

$$
\left[a_{1}, c_{1}\right] \otimes a_{1}=\left[a_{2}, c_{2}\right] \otimes a_{1}=\left(a_{2} \otimes c_{2}\right) \cdot{ }^{a_{1}}\left(a_{2} \otimes c_{2}\right)^{-1}=1_{\otimes} .
$$

For the other elements in $A$ the result follows in a similar manner. Since $W=\left\langle a_{1}, b_{1}, c_{1}, a_{2}, b_{2}, c_{2}\right\rangle$, we conclude $Z^{\otimes}(W)=\left\langle\left[a_{1}, b_{1}\right],\left[a_{1}, c_{1}\right]\right\rangle$ which is properly contained in $Z(W)$.

Finally, we compute $R_{2}^{\otimes}(W)$. Without further reference we shall use the fact that in all our calculations the commutator and tensor expansions are linear. This comes from the fact that $W$ has class 2 and thus we can apply Lemma 2.7. Consider $\left[a_{i}, h\right] \otimes h$ with $i=1,2$ and $h \in W$. By expansion $\left[a_{i}, h\right] \otimes h$ can be expressed as a product of factors of the form $\left[a_{i}, b_{i}\right] \otimes w$ and $\left[a_{i}, c_{i}\right] \otimes w$ and their inverses, where $w \in A$. By the relations of $W$ and (2.3.3) it follows that each of these factors equals one. We conclude that $\left[a_{i}, h\right] \otimes h=1_{\otimes}$, for all $h \in W$, and hence $a_{1}, a_{2} \in R_{2}^{\otimes}(W)$.

We claim now that $R_{2}^{\otimes}(W)=\left\langle a_{1}, a_{2}, Z(W)\right\rangle$. Every $w \in W$ can be written as $w=a_{1}^{\alpha_{1}} a_{2}^{\alpha_{2}} b_{1}^{\beta_{1}} b_{2}^{\beta_{2}} c_{1}^{\gamma_{1}} c_{2}^{\gamma_{2}} w^{\prime}$ with integers $\alpha_{1}, \alpha_{2}, \beta_{1}, \beta_{2}, \gamma_{1}, \gamma_{2}$ and $w^{\prime} \in W^{\prime}$. To prove our claim, it suffices to show that for every $w=b_{1}^{\beta_{1}} b_{2}^{\beta_{2}} c_{1}^{\gamma_{1}} c_{2}^{\gamma_{2}}$, with $\beta_{1}, \beta_{2}, \gamma_{1}, \gamma_{2}$ not all zero, there exists $h \in W$ such that $[w, h] \otimes h \neq 1_{\otimes}$. By expansion we obtain

$$
[w, h] \otimes h=\left(\left[b_{1}, h\right] \otimes h\right)^{\beta_{1}}\left(\left[b_{2}, h\right] \otimes h\right)^{\beta_{2}}\left(\left[c_{1}, h\right] \otimes h\right)^{\gamma_{1}}\left(\left[c_{2}, h\right] \otimes h\right)^{\gamma_{2}} .
$$

If $\beta_{1} \neq 0$ we choose $h=c_{1}$ and arrive at $[w, h] \otimes h=\left(\left[b_{1}, c_{1}\right] \otimes c_{1}\right)^{\beta_{1}} \neq 1_{\otimes}$, since by [1] we have that $\left[b_{1}, c_{1}\right] \otimes c_{1}$ is a nontrivial basis element of $W \otimes W$. Similarly, choosing 
$h=c_{2}, b_{1}$ or $b_{2}$ if $\beta_{2}, \gamma_{1}$ or $\gamma_{2}$ are not equal to zero, respectively, leads to [w, $\left.h\right] \otimes h \neq 1_{\otimes}$. We conclude that $R_{2}^{\otimes}(W)=\left\langle a_{1}, a_{2}, Z(W)\right\rangle$. Since $W=R_{2}(W)$, it follows that $R_{2}^{\otimes}(W)$ is a proper subgroup of $R_{2}(W)$, as claimed.

\section{REFERENCES}

1. M. Bacon, The nonabelian tensor square of a nilpotent group of class 2, Glasgow Math. J. 36 (1994), 291-296.

2. R. Brown, D. L. Johnson and E. F. Robertson, Some computations of nonabelian tensor products of groups, J. Algebra 111 (1987), 177-202.

3. R. Brown and J.-L. Loday, Excision homotopique en basse dimension, C.R. Acad. Sci. Paris Ser. I Math. 298 (1984), 353-356.

4. R. Brown and J.-L. Loday, Van Kampen theorems for diagrams of spaces, Topology 26 (1987), 311-335.

5. R. K. Dennis, In search of new "homology" functors having a close relationship to $K$-theory, Preprint, Cornell University, Ithaca, New York (1976).

6. Graham J. Ellis, Tensor products and $q$-crossed modules, J. London Math. Soc. (2) 51 (1995), 243-258.

7. T. Ganea, Homology at extensions centrales des groupes, C. R. Acad. Sci. Paris 266 (1968), 556-568.

8. P. Hall, Verbal and marginal subgroups, J. Reine Angew. Math. 182 (1940), 156-157.

9. L.-C. Kappe, Nonabelian tensor products of groups: the commutator connection, in Proceedings Groups - St. Andrews 1997 at Bath, London Math. Soc. Lecture Notes No 261 (1999), 447-454.

10. L.-C. Kappe and P. M. Ratchford, On centralizer-like subgroups associated with the n-Engel word, Algebra Colloq. 6 (1999), 1-8.

11. W. P. Kappe, Die A-Norm einer Gruppe, Illinois J. Math 5 (1961), 187-197.

12. A. S.-T. Lue, The Ganea map for nilpotent groups, J. London Math. Soc. (2) 14 (1976), 309-312.

13. I. D. Macdonald, Some examples in the theory of groups in Mathematical Essays Dedicated to A. J. MacIntyre (Ohio University Press, 1970), 263-269.

14. C. Miller, The second homology group of a group; relations among commutators, Proc. Amer. Math. Soc. 3 (1952), 588-595.

15. J. H. C. Whitehead, A certain exact sequence, Ann. of Math. (2) 52 (1950), 51-110. 